\title{
Cognición social en el abuso de sustancias. Una revisión narrativa
}

\author{
Jessica Zapata Téllez' \\ 'Departamento de Investigación Psicosocial y Documental, Centros de Integración Juvenil, A.C., México
}

\section{RESUMEN}

Introducción: la Cognición Social (CS) es el conjunto de procesos neurobiológicos, psicológicos y sociales que permiten percibir, reconocer y evaluar una situación social con el objetivo de generar la respuesta conductual más apropiada para cada contexto. Se integra por cinco dominios: Teoría de la Mente, Procesamiento Emocional, Estilo Atribucional, Percepción Social y Conocimiento Social. Al hablar del abuso de sustancias, la cs cobra relevancia por su implicación en el pronóstico terapéutico y la reinserción social de los usuarios en rehabilitación. Objetivo: explorar la información existente dentro de la literatura científica, en torno al funcionamiento de los dominios de CS en abusadores de sustancias. Método: se realizó una búsqueda de información en bases de datos digitales. Se consideraron investigaciones cuasi y experimentales, publicadas a partir del año 2000, que incluyeran la evaluación de al menos un dominio de la CS en participantes con abuso o dependencia de sustancias.

Resultados: se encontraron reportes de investigación que documentan alteraciones en los dominios de Procesamiento Emocional y Teoría de la Mente, en casos de abuso de alcohol, cannabis, metanfetaminas, cocaína y opiáceos; las alteraciones tienden a variar dependiendo de la sustancia y la severidad de consumo. No se encontraron documentos que evidencien el funcionamiento de los dominios Sesgo Atribucional, Percepción Social ni Conocimiento Social. Discusión y conclusiones: resulta aventurado señalar la existencia de un déficit de CS y sus dominios para cada sustancia en específico; los estudios son aún escasos, existe variabilidad respecto a los instrumentos empleados y falta considerar otras variables clínicas que pudieran moderar o modificar la asociación entre los dominios de la CS y el consumo abusivo de sustancias.

Palabras clave: Cognición Social, consumo de sustancias, Teoría de la Mente, Procesamiento Emocional.

\begin{abstract}
Introduction: Social Cognition (CS, for its acronym in Spanish) is the set of neurobiological, psychological and social processes that allow to perceive, recognize and asses a social situation to generate the most appropriate behavioral response for each context. It is comprised by five domains: Theory of Mind, Emotional Processing, Atributional Bias, Social Perception and Social Knowledge. On regard of substance abuse, the SC becomes relevant for their involvement in the therapeutic prognosis and social reintegration of drug users in rehabilitation. Objective: to explore existing information within the scientific literature related to the functioning of CS domains in substance abusers. Method: a search in digital databases was carried out. Quasi and experimental researches, published since 2000, which included the evaluation of at least one CS domain in subjects with substance abuse or dependence, were considered. Results: research reports documenting alterations in Emotional Processing and Theory of Mind associated with the abuse of alcohol, cannabis, methamphetamines, cocaine, and opiates. Alterations may vary depending on the substance and severity of consumption. No publications were found that demonstrate the performance of Attribution Bias, Social Perception or Social Knowledge. Discussion: it is risky to point out the existence of a deficit in CS domains, for each specific substance; studies are still scarce, there is variability with respect to the instruments used, and there are other clinical variables that could moderate or modify the association between the domains of CS and substance abuse.
\end{abstract}

Keywords: Social Cognition, substance abuse, Theory of Mind, Emotional Processing.

\footnotetext{
Autor de correspondencia:

Jessica Zapata Téllez. Centros de Integración Juvenil, A.C., Tlaxcala núm. 208, col. Hipódromo, del. Cuauhtémoc, C.P. 06100, Ciudad de México, México. Tel. +52(55) 5999 7760. Correo electrónico: psic.zapata@gmail.com

Recibido: 18 de noviembre de 2016

Aceptado: 21 de abril de 2017

DOI: 10.28931/riiad.2017.1.06
} 


\section{INTRODUCCIÓN}

Los trastornos por consumo de sustancias son patrones problemáticos que no cesan a pesar del deterioro o malestar físico y psicológico clínicamente significativo que provocan. Suelen caracterizarse por un poderoso deseo o necesidad de consumir, en cantidades cada vez mayores, para alcanzar el efecto deseado; llevan a invertir una gran cantidad de tiempo en actividades asociadas con la sustancia: conseguirla, consumirla o recuperarse de sus efectos; y pueden provocar riesgos físicos, incumplimiento de deberes y abandono de actividades sociales, profesionales o de ocio (Asociación Psiquiátrica Americana, 2014).

Dentro del extenso abanico de consecuencias negativas para la salud asociadas con el consumo frecuente y prolongado de sustancias, se encuentran las de índole neurocognitivo (Bolla et al., 2003), presentes en entre 30 y $80 \%$ de los afectados por estos trastornos que, según la evidencia, presentan alteraciones de magnitud variable (Bates, Pawlak, Tonigan, \& Buckman, 2006) en diversos dominios y diferenciadas por grupos de sustancias.

Los psicoestimulantes, por ejemplo, han sido asociados con déficits en ciertas funciones ejecutivas, como la inhibición conductual y la flexibilidad cognitiva; el alcohol, con déficits en la inhibición conductual, la flexibilidad cognitiva, el procesamiento espacial, la velocidad perceptiva y la atención selectiva; el MDMA, con déficits en el procesamiento espacial, la velocidad perceptiva y de procesamiento, la atención selectiva y la planeación; el cannabis, con déficits en la memoria prospectiva, la velocidad de procesamiento y la planificación; y las metanfetaminas, con la afectación de la memoria prospectiva (Fernández-Serrano, Pérez, \& Verdejo, 2011; Rosenberg, Grigsby, Dreisbachb, Busenbark, \& Grigsby, 2002; Salo, Ursu, Buonocore, Leamon, \& Carter, 2009; Vik, Celluci, Jarchow, \& Hedt, 2004).

La relevancia de estos déficits radica en sus implicaciones clínicas, pues se ha puesto en evidencia que dificultan la participación en tareas terapéuticas que requieren, entre otras cuestiones, la integridad de habilidades involucradas con el aprendizaje, ya que se espera que los afectados reciban, codifiquen e integren información novedosa que pueda traducirse en el establecimiento y el mantenimiento de estrategias conductuales más adaptativas (Goldman, 1990; Weinstein \& Shaffer, 1993).

Ahora bien, existe otro cúmulo de alteraciones, como el razonamiento social, la toma de decisiones, la dificultad para entablar y mantener relaciones sociales y para otorgar valor a las consecuencias adversas, que también han sido observadas en estos trastornos y pertenecen a otro dominio de la neurocognición identificado con el nombre de Cognición Social (CS) (Goldstein \& Volkow, 2002). Estas alteraciones resultan relevantes, pues influyen en la percepción, la interpretación y la respuesta a los estímulos socio-emocionales, cuestión de enorme importancia para la adaptación social de los afectados por trastornos asociados con el consumo de sustancias (Tirapú-Ustárriz, Pérez, Erekatxo-Bilbao, \& Pelegrín-Valero, 2007), en quienes el cese del consumo suele ser tan sólo el primer paso para una rehabilitación integral (Rodríguez \& Nute, 2013).

\section{La Cognición Social}

Desde las neurociencias cognitivas, la CS se define como un conjunto de procesos neurobiológicos (Butman, 2001) y psicológicos que permiten percibir, reconocer y evaluar una situación social para construir una representación del ambiente de interacción y, posteriormente, llevar a cabo la respuesta conductual más apropiada para el contexto (Adolphs, 2001). Al respecto, el Instituto Nacional de Salud Mental de Estados Unidos ha planteado una estructura de CS compuesta por cinco dominios que se resumen en la Tabla 1 (Green et al., 2008).

De acuerdo con las teorías explicativas sobre el desarrollo de estas habilidades, existe de manera innata un conjunto de mecanismos con bases neurales fijas especializadas en el procesamiento y la representación de información específica para cada dominio de la CS (Rowe et al., 2007), pero, a decir de algunos teóricos, estos módulos no se encuentran activos desde el nacimiento, pues es necesario que converjan ciertos precursores (como otras habilidades cognitivas innatas o el desarrollo de otros circuitos neurales), que junto con la experiencia social desencadenen estos mecanismos y propicien su evolución (Aguirre, 2013; Pascual, Aguado, \& Sotillo, 2006).

Respecto a estas bases neurales, se ha logrado identificar una compleja red de conexiones distribuidas por todo el cerebro, mismas que al coordinarse e interactuar permiten que las habilidades de CS se expresen. En la Tabla 2 se señalan las principales estructuras asociadas con los diferentes dominios de cognición social (Bechara, 2002; Butman, 2001; Gil, 2007; Sugranyes, Kyriakopoulos, Corrigall, Taylor, \& Frangou, 2011). La evidencia sugiere que cualquier daño o alteración en la química cerebral de estas estructuras puede reflejar dificultades en las habilidades de CS (España, 2013): Ias lesiones en el giro fusiforme derecho y en la amígdala, por ejemplo, pueden dan lugar a una incapacidad para reconocer las expresiones faciales de las emociones (Adolphs, 2009; Barton, 2008), mientras que las lesiones en la amígdala y los lóbulos temporales se reflejarían en la incapacidad 
de adecuar la expresión de las emociones a un contexto en particular (Adolphs, 2010).

Entre las condiciones asociadas con déficits en los dominios de CS se encuentran los trastornos del espectro autista, los trastornos de personalidad y la esquizofrenia, entre otros, en los que se han documentado alteraciones neuroanatómicas y funcionales de base (Aedo, Waissbluth, Kunstman, \& Botto, 2010; Damasio, Tranel, \& Damasio, 1990; Dapretto et al., 2005); pero también están aquellas en las que se observa un deterioro de estos dominios cuando ya han alcanzado cierto nivel de desarrollo, como la epilepsia severa, los traumatismos craneoencefálicos, las demencias, las intervenciones neuroquirúrgicas y el abuso de sustancias (Adolphs, 2001; Eslinger \& Damasio, 1985; España, 2013).

En consideración de esto, la presente revisión se plantea el objetivo de explorar la información existente dentro la literatura científica en torno al funcionamiento de los dominios de CS en abusadores de sustancias.

\section{METODOLOGÍA}

Durante el segundo trimestre de 2016, se realizó una búsqueda de información en las siguientes bases de datos electrónicas: EBSCO, ScienceDirect, SCOPUS y SCIELO; se usaron los términos de búsqueda "Cogni-

Tabla 1

Dominios de la Cognición Social

\begin{tabular}{|c|c|}
\hline Dominio & Definición \\
\hline Teoría de la Mente & $\begin{array}{l}\text { Habilidad heterometacognitiva que permite inferir el estado mental (intenciones, deseos, disposicio- } \\
\text { nes, pensamientos, etcétera) de otros. }\end{array}$ \\
\hline $\begin{array}{l}\text { Procesamiento } \\
\text { Emocional }\end{array}$ & $\begin{array}{l}\text { Habilidad para percibir y expresar las emociones; incluye cuatro componentes: reconocimiento, } \\
\text { facilitación, comprensión y manejo emocionales. }\end{array}$ \\
\hline $\begin{array}{l}\text { Sesgo } \\
\text { Atribucional }\end{array}$ & $\begin{array}{l}\text { El Sesgo o Estilo Atribucional refleja la forma en la que una persona infiere o explica las causas o los } \\
\text { resultados de un evento determinado, ya sea positivo o negativo; incluye tres componentes: interna- } \\
\text { lidad/externalidad, estabilidad/inestabilidad y globalidad/especificidad. }\end{array}$ \\
\hline Percepción Social & $\begin{array}{l}\text { Habilidad para identificar los componentes que caracterizan las relaciones interpersonales (los roles } \\
\text { sociales, el nivel de intimidad, la veracidad, el estatus social, la naturaleza de las relaciones entre } \\
\text { personas, la metas, etcétera). }\end{array}$ \\
\hline Conocimiento Social & $\begin{array}{l}\text { Habilidad para identificar los componentes que caracterizan determinadas situaciones sociales (los } \\
\text { contextos, las normas y reglas sociales, las expectativas). }\end{array}$ \\
\hline
\end{tabular}

Tabla 2

Principales estructuras cerebrales implicadas en las habilidades de CS

\begin{tabular}{|c|c|c|c|c|c|}
\hline Estructura & ToM & PE & SA & PS & CS \\
\hline Surco temporal superior & $\checkmark$ & $\checkmark$ & & & \\
\hline Corteza prefrontal medial & $\checkmark$ & $\checkmark$ & & & $\checkmark$ \\
\hline Corteza prefrontal ventromedial & $\checkmark$ & $\checkmark$ & $\checkmark$ & $\checkmark$ & $\checkmark$ \\
\hline Corteza prefrontal dorsomedial & $\checkmark$ & $\checkmark$ & & $\checkmark$ & $\checkmark$ \\
\hline Corteza orbitofrontal & & & & $\checkmark$ & $\checkmark$ \\
\hline Corteza somatosensorial & & & & $\checkmark$ & $\checkmark$ \\
\hline Amígdala & & $\checkmark$ & & & $\checkmark$ \\
\hline Girofusiforme & & $\checkmark$ & $\checkmark$ & & $\checkmark$ \\
\hline Ínsula & $\checkmark$ & $\checkmark$ & $\checkmark$ & & \\
\hline Cíngulo & & & $\checkmark$ & & $\checkmark$ \\
\hline Cortex frontal & $\checkmark$ & & & & \\
\hline Unión temporo-parietal & & & $\checkmark$ & $\checkmark$ & \\
\hline
\end{tabular}

ToM = Teoría de la Mente; PE = Procesamiento Emocional; SA = Sesgo Atribucional; PS = Percepción Social;

$\mathrm{CS}=$ Conocimiento Social. 
ción Social/Social Cognition", "Teoría de la Mente/Mind Theory-Theory of Mind", "Procesamiento Emocional/ Emotional Processing", "reconocimiento facial de emociones/facial emotion recognition", "empatía/empathy", "Conocimiento Social/Social Knowledge" y "Percepción Social/Social Perception"; mediante el empleo del conector "y/and", los términos se combinaron con "abuso de sustancias/substance abuse", "alcohol/alcohol", "cocaína/cocaine", "cannabis/cannabis", "inhalantes/inhalants", "estimulantes/stimulants drugs", "alucinógenos/ hallucinogens", "drogas depresoras/depressant drugs" y "opiáceos/opiates".

Se consideraron reportes de investigación cuasi-experimentales y experimentales desarrollados bajo la perspectiva neurocognitiva, publicados en revistas científicas indexadas a partir del año 2000 (año aproximado en el que la investigación en CS bajo esta perspectiva comenzó a proliferar) y escritos en español o inglés; se buscó, además, que incluyeran la evaluación de al menos un dominio de la CS en participantes con abuso o dependencia de sustancias. La búsqueda se com-

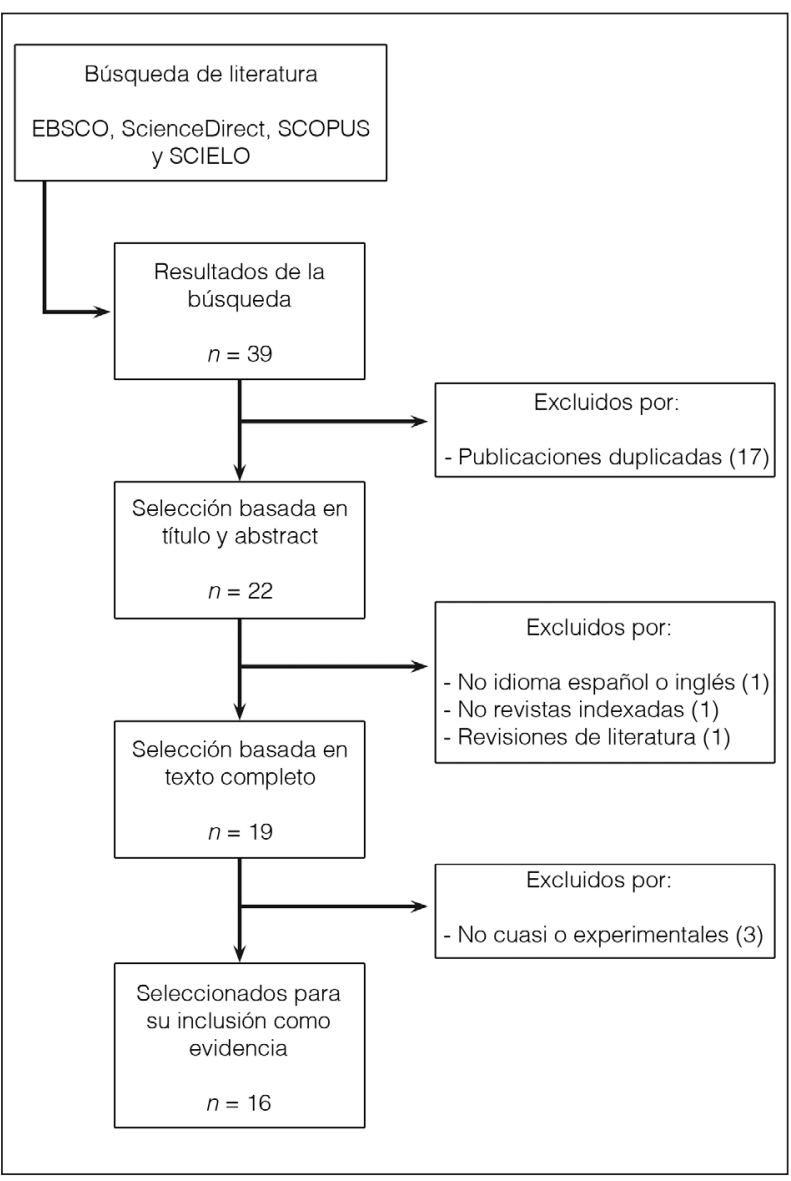

Figura 1. Flujograma de la revisión de literatura plementó con la consulta de las listas de referencias de los artículos primarios. Debido al interés por realizar una revisión meramente exploratoria y descriptiva en torno a la evidencia sobre el tema, no se establecieron otros criterios de inclusión o exclusión a priori.

\section{RESULTADOS}

Se incluyeron un total de 16 publicaciones científicas que cumplieron con las características especificadas. Se encontró evidencia únicamente para dos dominios de la CS: Procesamiento Emocional y Teoría de la Mente. No se encontraron publicaciones que cumplieran con las características establecidas y que evaluaran los dominios Sesgo Atribucional, Conocimiento Social y Percepción Social. En el Anexo 1 se detallan las características de los estudios incluidos en esta revisión. Una vez integrada toda la información, se determinó organizar la evidencia encontrada en los siguientes apartados clasificados por sustancia de abuso: a) Procesamiento Emocional y b) Teoría de la Mente.

\section{Procesamiento Emocional}

\section{Procesamiento Emocional y abuso de sustancias}

Se incluyeron, para su revisión, dos artículos de índole cuantitativo, cuasi experimentales, con grupos comparativos y mediciones transversales. La evaluación de este dominio se llevó a cabo mediante índices de respuesta (Valencia, Activación y Dominancia) ante estímulos afectivos y mediante el reconocimiento facial de emociones. Los tamaños de muestra oscilaron entre 44 y 64 participantes por grupo e incluyeron consumidores de sustancias en general y participantes sin antecedentes de problemas asociados con el consumo.

En estos estudios se encontró que tanto los usuarios de sustancias como los no consumidores perciben los estímulos afectivos con contenido sexual como más agradables, mientras que los que tienen contenido aversivo son considerados como los más desagradables. Sin embargo, los consumidores tienden a sobrevalorarlos de manera más extrema (Chicharro, Pérez-García, \& Sanjuán, 2011); es decir, que valoran los estímulos agradables como mejores y los desagradables como peores.

Respecto al reconocimiento facial de emociones, se ha encontrado que los consumidores de sustancias presentan problemas para identificar correctamente las expresiones faciales emocionales, en especial las de ira, asco, miedo y tristeza. Para estas dos últimas, la apatía se ha encontrado asociada de manera inversamente proporcional (Fernández-Serrano, Moreno, Pérez, \& Verdejo, 2012), es decir, que mientras menos apatía se 
Tabla 3

Drogas y dominios de la CS evaluados en los artículos seleccionados como evidencia

\begin{tabular}{|c|c|c|c|c|c|c|c|c|}
\hline \multirow[t]{2}{*}{ Autores } & \multicolumn{2}{|c|}{ Dominio CS } & \multicolumn{6}{|c|}{ Droga } \\
\hline & PE & ToM & ALC & CAN & MET & $\mathrm{COC}$ & OPI & POL \\
\hline Kornreich et al. (2001) & $\checkmark$ & & $\checkmark$ & & & & & \\
\hline Kornreich et al. (2002) & $\checkmark$ & & $\checkmark$ & & & & & \\
\hline Townshend \& Duka (2002) & $\checkmark$ & & $\checkmark$ & & & & & \\
\hline Foisy et al. (2007) & $\checkmark$ & & $\checkmark$ & & & & & \\
\hline Kemmis et al. (2007) & $\checkmark$ & $\checkmark$ & & & & $\checkmark$ & & \\
\hline Woicik, Moeller, \& Alia-Klein (2009) & $\checkmark$ & & & & & $\checkmark$ & & \\
\hline Fernández-Serrano et al. (2010) & $\checkmark$ & & & & & & & $\checkmark$ \\
\hline Aguilar de Arcos et al. (2011) & $\checkmark$ & & $\checkmark$ & & & $\checkmark$ & & \\
\hline Chicharro et al. (2011) & $\checkmark$ & & & & & & & $\checkmark$ \\
\hline Kim et al. (2011) & $\checkmark$ & $\checkmark$ & & & $\checkmark$ & & & \\
\hline Fernández-Serrano et al. (2012) & $\checkmark$ & & & & & $\checkmark$ & & \\
\hline Martín-Contero et al. (2012) & $\checkmark$ & & & & & & $\checkmark$ & \\
\hline Roser et al. (2012) & & $\checkmark$ & & $\checkmark$ & & & & \\
\hline Bosco et al. (2014) & & $\checkmark$ & $\checkmark$ & & & & & \\
\hline Ersche et al. (2015) & $\checkmark$ & & & & & $\checkmark$ & & \\
\hline Kuypers et al. (2015) & $\checkmark$ & & & & & $\checkmark$ & & \\
\hline Maurage et al. (2015) & & $\checkmark$ & $\checkmark$ & & & & & \\
\hline
\end{tabular}

$\mathrm{PE}=$ Procesamiento Emocional; ToM = Teoría de la Mente; ALC = alcohol; CAN = cannabis; MET = metanfetaminas;

COC = cocaína; POL = polisustancias .

presente, el reconocimiento del miedo y la tristeza tiende a mejorar. Además, se ha reportado que el consumo de sustancias puede ser un buen predictor de las fallas en el reconocimiento de las expresiones faciales correspondientes a la ira (Fernández-Serrano, Pérez-García, \& Verdejo-García, 2011).

\section{Procesamiento Emocional y abuso de alcohol}

Se incluyeron cinco artículos, todos ellos de índole cuantitativo, cuasi experimentales, con grupos comparativos y mediciones transversales. La evaluación se hizo mediante índices de respuesta (Valencia, Activación y Dominancia) ante estímulos afectivos y mediante el reconocimiento facial de emociones. Los tamaños de muestra oscilaron entre 14 y 30 participantes por grupo, en los que se incluyeron personas hospitalizadas por dependencia del alcohol, consumidores con distintos patrones de tratamiento, usuarios de otras sustancias y participantes sin antecedentes psiquiátricos o sin problemas con el consumo de alcohol.

Al respecto, se ha reportado que los participantes consumidores que se encuentran en periodo de abstinencia de por lo menos dos meses presentan mayores dificultades que los sanos para reconocer el asco y la ira (Kornreich et al., 2001). Por su parte, los pacientes en reciente desintoxicación, con hasta tres semanas de abstinencia, muestran mayores dificultades que los sanos para reconocer las expresiones faciales (Kornreich et al., 2002), en especial las del miedo (Townshend \& Duka, 2002) y la tristeza (Foisy et al., 2007), y suelen confundir, en mayor medida que los participantes sanos, las expresiones de alegría con las de sorpresa y las de asco o enojo con las de ira o tristeza (Foisy et al., 2007).

Los pacientes en reciente desintoxicación también suelen sobrevalorar, en mayor medida que los partici- 
pantes sanos, la intensidad de las expresiones faciales de asco, ira, tristeza, sorpresa y miedo (Kornreich et al., 2001; Townshend \& Duka, 2002); además, requieren de más tiempo para reconocer una emoción y evaluar su intensidad (Foisy et al., 2007) y suelen presentar una mayor cantidad de problemas interpersonales que los participantes sanos (Kornreich et al., 2002).

Se ha observado que estos déficits suelen ser más intensos en personas con un mayor número de desintoxicaciones previas (Townshend \& Duka, 2002) y no difieren al incrementarse el tiempo en abstinencia, lo que sugiere que el déficit en reconocimiento facial de emociones en participantes con dependencia del alcohol se debe, en mayor medida, al consumo crónico y no al efecto de la sustancia misma (Kornreich et al., 2001); ambos hechos son congruentes con los cambios observados en el funcionamiento de la amígdala en dependientes del alcohol (Townshend \& Duka, 2002).

Por otra parte, y contrario a lo que podría esperarse, se ha reportado que los participantes con dependencia del alcohol que se encuentran en abstinencia controlada bajo tratamiento en régimen de internamiento tienden a presentar una menor capacidad perceptiva de estímulos emocionales, que los participantes con dependencia de cocaína, sobre todo en aquellos estímulos con valencias desagradables, niveles de activación elevados y dominancia baja, y aquellos con valencias agradables, niveles de activación alta y dominancias bajas (Aguilar de Arcos, Montañez, Gómez, \& Pérez-García, 2011).

\section{Procesamiento Emocional y abuso de cocaína}

Se incluyeron para su revisión cuatro reportes de investigación, todos ellos de índole cuantitativo, con grupos de comparación y mediciones transversales. Los tamaños de muestra oscilaron entre 15 y 48 participantes por grupo, en los que se incluyeron personas con dependencia de la cocaína, otras con diferentes patrones de consumo y no consumidores de drogas ilegales. Las evaluaciones se llevaron a cabo mediante pruebas de reconocimiento facial de emociones e inteligencia emocional.

Los resultados mostraron que los participantes que usan cocaína de manera recreativa presentan mayores dificultades que los consumidores ocasionales y que los no consumidores para reconocer las expresiones faciales emocionales, en especial las correspondientes al miedo, que tienden a ser confundidas con las de sorpresa; se observó que estas diferencias se vuelven más marcadas cuando se suma el consumo de cannabis (Kemmis, Hall, Kingston, \& Morgan, 2007). Además, los usuarios recreativos suelen requerir una mayor cantidad de tiempo que los no consumidores para diferenciar de manera correcta las expresiones faciales de las emocio- nes en general y tienden a requerir más tiempo que los usuarios ocasionales y los no consumidores para reconocer las expresiones de ira, miedo, alegría y sorpresa. Cuando se suma el consumo frecuente de alcohol, estas diferencias tienden a ser más marcadas para las expresiones de tristeza (Kemmis et al., 2007).

En abusadores crónicos de cocaína existe también un déficit, respecto a los no consumidores, en el reconocimiento de expresiones faciales de miedo, que se tiende a confundir con la sorpresa e incluso con la tristeza. Este efecto podría estar mediado por el coeficiente intelectual. Asimismo, se encontró que estos consumidores tienden a cometer una mayor cantidad de errores en el reconocimiento de las expresiones de ira, lo cual podría estar mediado por el uso concomitante de opiáceos (Ersche et al., 2015).

Bajo intoxicación, los consumidores recreativos suelen valorar las expresiones faciales de ira y tristeza con mayor intensidad que los usuarios recreativos no intoxicados; además, requieren de más tiempo para valorar las expresiones faciales de asco. Cuando el efecto de la droga disminuye en los sujetos intoxicados, el reconocimiento de las expresiones de tristeza empeora notablemente; mientras que para las expresiones de ira y asco, el reconocimiento mejora, equiparándose incluso, al de los consumidores no intoxicados (Kuypers, Steenberger, Toennes, \& Ramaekers, 2015). Se ha reportado también que la duración del consumo de cocaína puede ser un buen predictor del déficit en el reconocimiento de expresiones faciales correspondientes a la ira y el miedo (Fernández-Serrano et al., 2011).

Respecto a los pacientes con dependencia que se encuentran en abstinencia, se ha reportado que tienden a presentar una menor capacidad que los no consumidores para enfrentarse a situaciones estresantes de una manera adaptativa, así como una menor capacidad de pensar y generar conductas eficaces; muestran, de igual forma, una mayor tendencia a dicotomizar, categorizar, simplificar y prejuiciar, con lo que evidencian una menor inteligencia experiencial y emocional (Fernández-Serrano et al., 2012).

Los investigadores señalan que los resultados encontrados no pueden explicarse por los efectos subagudos e inmediatos de la cocaína, pues tanto los consumidores con inicio reciente como los de uso crónico presentan déficits similares al ser comparados con participantes no consumidores. En este sentido, los estudios señalan la importancia de considerar los posibles paralelismos entre los consumidores regulares de cocaína y los rasgos sociopáticos de la personalidad, ya que en ambos casos existe una dificultad para reconocer expresiones emocionales de miedo y una disfunción amigdalar (Kemmis et al., 2007). 


\section{Procesamiento Emocional y abuso de metanfetaminas}

Se incluyó un artículo en el que se evalúa este dominio mediante el reconocimiento facial de emociones. Es una investigación de índole cuantitativo, transversal y con diseño de casos y controles en el que se incluyeron abusadores de metanfetaminas y controles sanos, con un tamaño de muestra aproximado de 28 participantes por grupo. La evaluación se realizó mediante índices de respuesta (Valencia, Activación y Dominancia) ante estímulos afectivos.

Se reportó que los usuarios de metanfetaminas tienden a presentar mayores dificultades que los no consumidores para reconocer las expresiones faciales de las emociones, en especial las correspondientes al miedo. También se encontró que los usuarios tienden a ser menos flexibles cognitivamente, que aquellos participantes no consumidores, encontrando además que este déficit de flexibilidad cognitiva se asocia de manera directa con la habilidad para reconocer las expresiones faciales emocionales (Kim, Kwon, \& Chang, 2011).

\section{Procesamiento Emocional y abuso de opiáceos}

Fue incluido un artículo de índole cuantitativo, trasversal con dos grupos comparativos, en el que se evalúa el Procesamiento Emocional mediante el reconocimiento facial de emociones y la empatía. Se incluyeron 18 pacientes con dependencia de opiáceos y 18 participantes sin presencia, pasada o actual, de abuso o dependencia de drogas.

Se documentó que los consumidores no muestran diferencias con los no consumidores, respecto a la inteligencia emocional o al reconocimiento facial de emociones. Sin embargo, sí presentan menos habilidades inter e intrapersonales, menor capacidad adaptativa, de tolerancia y manejo del estrés, menor autoconsideración y autoactualización, menor prueba de realidad, peor humor general, menor sensación de felicidad y menor capacidad para manifestar empatía en general, al ser comparados con participantes no consumidores (Martín-Contero, Secades-Villa, \& Tirapu-Ustárroz, 2012).

\section{Teoría de la Mente}

\section{Teoría de la Mente y abuso de alcohol}

Se consideraron dos reportes de investigación en los que se evaluó este dominio, ambos de índole cuantitativo, transversal y con grupos comparativos en los que se incluyeron tanto participantes abusadores como sin problemas con el alcohol. Las muestras oscilaron entre 22 y 34 personas por grupo.
Se ha encontrado que los consumidores en reciente desintoxicación tienden a presentar problemas mucho más serios que los participantes sin dificultades con el alcohol para responder diversas pruebas de inteligencia; además, presentan síntomas de ansiedad y depresión más severos. También se ha reportado que estos consumidores muestran complicaciones al hacer uso de las habilidades metacognitivas, lo que les dificulta evaluar las situaciones sociales con toma de perspectiva y, por ende, detectar los engaños y las falsas creencias (Maurage, Timary, Tecco, Lechantre, \& Samson, 2015). Cuando se encuentran en un periodo de abstinencia prolongado siguen presentando estas complicaciones, pero también serios problemas para comprender y atribuir las intenciones, los deseos y las creencias, tanto en la subescala Primera Persona -en la que se evalúa la capacidad para conocer y comprender los propios estados mentales-, como en la de Tercera Persona -en la cual se mide la capacidad para conocer y comprender los estados mentales de otras personas cuando se manifiestan en primera o en tercera persona-; cuestión que, se ha visto, tiende a empeorar con el abuso crónico del alcohol (Bosco, Capozzi, Colle, Marostica, \& Tirassa, 2014).

De acuerdo con los investigadores, estos déficits pueden vincularse con las características del patrón de uso, como su duración, el consumo promedio e, incluso, el deseo por consumir. Aunque estas complicaciones son compatibles con una disfunción de la corteza prefrontal (Bosco et al., 2014), también han sido asociadas con lesiones en el área temporo-parietal, lo que indicaría que los déficits sociales observados en la dependencia del alcohol no tienen un origen neuroanatómico único (Maurage et al., 2015).

\section{Teoría de la Mente y abuso de cannabis}

Fue incluido un solo artículo, de índole cuantitativo, transversal y con grupos comparativos que incluyeron a 15 participantes consumidores crónicos y a 14 participantes sin dependencia por consumo de sustancias.

Los resultados muestran que los consumidores crónicos, en abstinencia de 24 horas, tienden a presentar problemas para entender las indirectas y captar las falsas creencias de las otras personas. Además, durante las tareas de mentalización activan regiones cerebrales distintas a los no consumidores, quienes muestran focos de activación en regiones prefrontales bilaterales y temporoparietales, en el cíngulo anterior y posterior y en la ínsula lateral derecha; mientras que los consumidores crónicos presentan focos de activación en el cíngulo posterior derecho, precúneo bilateral y regiones temporoparietales. Aunado a estas diferencias, los consumido- 
res crónicos de cannabis también muestran una menor prominencia de activación que los no consumidores, en el giro para-hipocampal izquierdo y el precúneo lateral derecho; pero una mayor prominencia en el precúneo lateral izquierdo y el giro cingular anterior derecho (Roser et al., 2012).

De acuerdo con los autores, los patrones de activación cerebral presentados por los consumidores crónicos de cannabis se asemejan a los encontrados en muestras de participantes con riesgo a desarrollar trastornos del espectro psicótico (Roser et al., 2012), quienes tienden a sobrementalizar o sobreinterpretar los estados mentales de las otras personas. Esto sugiere que el consumo crónico de cannabis podría afectar el procesamiento de la información social de manera similar a otros factores de riesgo.

\section{Teoría de la Mente y abuso de metanfetaminas}

Se consideró un reporte de investigación de índole cuantitativo, transversal y con dos grupos de comparación que incluyeron 28 hombres con abuso de metanfetaminas y 27 sin diagnóstico psiquiátrico alguno.

Según los resultados, los dependientes de la cocaína con al menos una semana de abstinencia tienden a presentar dificultades para comprender e interpretar el estado de otras personas a través de su rostro. Ya que, además de presentar problemas en el reconocimiento facial de emociones, les resulta complicado inferir de manera correcta los deseos, las intenciones o los pensamientos a través de la mirada. Asimismo, presentan un déficit importante en el desciframiento de las insinuaciones e indirectas de otras personas y se muestran perseverantes a pesar de los errores (Kim et al., 2011).

Al respecto, los investigadores señalan la importancia de considerar, en el tratamiento y la rehabilitación de estos consumidores, el papel del reconocimiento facial de emociones y la Teoría de la Mente, así como de trabajar en el diseño de intervenciones dirigidas a recuperar y mejorar las interacciones sociales (Kim et al., 2011).

\section{DISCUSIÓN Y CONCLUSIONES}

El objetivo de esta revisión narrativa fue explorar la información existente dentro la literatura científica, en torno al funcionamiento de los dominios de CS en abusadores de sustancias. En la búsqueda no se encontró evidencia que cumpliera con las características establecidas en esta investigación y que abordara los dominios Sesgo Atribucional, Conocimiento Social y Percepción Social, por lo que resulta imposible afirmar la existencia de un déficit de CS asociado con el abuso de drogas.
Este hecho es congruente con lo encontrado en otras patologías, en las que estos dominios han sido, también, los estudiados con menor frecuencia, lo que quizá esté relacionado con la falta de instrumentos específicos, estandarizados y validados, que permitan evaluar estos dominios; en ello identificamos un área de oportunidad para futuras investigaciones.

A pesar de esto, la evidencia encontrada permite vislumbrar serias dificultades en las habilidades de Procesamiento Emocional y Teoría de la Mente asociadas con el abuso y la dependencia de diversas sustancias, problemas que tienden a variar en cualidad y severidad dependiendo de la sustancia y el patrón de consumo. Resulta aventurado afirmar que estas dificultades forman parte de un déficit específico en estos dominios, pues los resultados no han sido sistemáticos ni concluyentes.

Esto está probablemente asociado con la presencia de factores confusores que podrían estar impactando sobre estos dominios y que no han sido evaluados en todos los estudios; con esto identificamos otra área de oportunidad que llama al diseño de estrategias metodológicas controladas que incluyan el control de variables identificadas en la literatura como confusoras (tratamiento psicofarmacológico, enfermedades neurológicas, traumatismos craneoencefálicos, enfermedades psiquiátricas comórbidas o trastornos del desarrollo en la infancia, trastornos de personalidad, funcionamiento cognitivo, episodios psicóticos, abuso de múltiples sustancias y nivel educativo, entre otras) y que contemplen criterios de inclusión más estrictos que permitan establecer patrones de disfunción en Cs específicos para cada sustancia.

Se pueden resumir varios puntos identificados en este estudio: para el Procesamiento Emocional suelen usarse con mayor frecuencia las pruebas de reconocimiento facial de emociones de Ekman, en las que se requiere realizar dos tareas, reconocer una emoción y evaluar su intensidad. Al respecto, los abusadores de alcohol tienden a presentar problemas para reconocer la ira, el asco y la tristeza, y tienen a sobrevalorar el miedo. Los consumidores de cocaína presentan dificultades para diferenciar el asco, el miedo y la sorpresa. Los policonsumidores presentan dificultades para reconocer la ira, el asco, el miedo y la tristeza; además, valoran los estímulos emocionales de forma más extrema. Los consumidores de metanfetaminas y de opiáceos presentan dificultades generales para reconocer una emoción.

La evidencia anterior es, sin duda, prueba del estado en el que se encuentra el estudio de la CS en el contexto de las adicciones; un campo novedoso y reciente con cuestiones abiertas y hallazgos algunas veces contradictorios. Esto, a su vez, es muestra de la necesidad de continuar aportando información que permita diseñar 
estrategias de intervención que apunten a mejorar otro factor importante dentro de la rehabilitación y la reinserción del sujeto adicto.

\section{Limitaciones}

No se encontraron estudios que cumplieran con las características planteadas en este estudio y que documentaran el funcionamiento de los dominios Sesgo Atribucional, Conocimiento Social y Percepción Social. El año establecido como punto de partida para realizar la búsqueda redujo la cantidad de evidencia que pudo haber sido incluida en el estudio. Los resultados presentados en esta revisión deben ser tomados con cautela, puesto que la evidencia seleccionada muestra sus propias limitaciones.

\section{REFERENCIAS}

Adolphs, R. (2001). The neurobiology of social cognition. Current Opinion in Neurobiology, 11(2), 231-239. doi: 10.1016/S09594388(00)00202-6

Adolphs, R. (2009). The social brain: neural basis of social knowledge. Annual Review of Psychology, 60(1), 693-716. doi: 10. 1146/annurev.psych.60.110707.163514

Adolphs, R. (2010). What does the amygdala contribute to social cognition? Annals of the New York Academy of Science, 1191(1), 4261. doi: 10.1111/j.1749-6632.2010.05445.x

Aedo, A., Waissbluth, D., Kunstmann, M., \& Botto, A. (2010). Neuronas espejo, mentalización y psicopatología. Gaceta de Psiquiatría Universitaria, 6(3), 309-316.

Aguilar de Arcos, F., Montañez, M., Gómez, E., \& Pérez-García, M. (2011). Influencia del contenido emocional en la percepción de estímulos visuales en sujetos drogodependientes. Trastornos Adictivos, 13(2), 57-63. doi: 10.1016/S1575-0973(11)70012-5

Aguirre, R. (2013) Desarrollo de la Cognición Social en personas con trastornos del espectro autista. Revista Chilena de Terapia Ocupacional, 13(2), 11-19.

Asociación Psiquiátrica Americana (2014). Manual Diagnóstico y Estadístico de los Trastornos Mentales. Barcelona: Masson.

Barton, J. J. (2008). Structure and function in acquired prosopagnosia: lesson from a series of 10 patients with brain damage. Journal of neuropsychology, 2(1), 197-225. doi:10.1348/174866407X214172

Bates, M. E., Pawlak, A. P., Tonigan, J. S., \& Buckman, J. F. (2006). Cognitive impairment influences drinking outcome by altering therapeutic mechanisms of change. Psychology of Addictive Behaviors, 20(3), 241-253. doi: 10.1037/0893-164X.20.3.241

Bechara, A. (2002). The neurology of social cognition. Brain, 125(8), 1673-1675. doi: 10.1093/brain/awf169

Bosco, F., Capozzi, F., Colle, L., Marostica, P., \& Tirassa, M. (2014). Theory of mind deficit in subjects with alcohol use disorder: an analysis of mind reading processes. Alcohol and Alcoholism, 49(3), 299-307. doi: 10.1093/alcalc/agt148
Bolla, K. I., Eldreth, D. A., London, E. D., Kiehl, K. A., Mouratidis, M., Contoreggi, C., ... Ernst, M. (2003). Orbitofrontal cortex dysfunction in abstinent cocaine abusers performing a decision-making task. Neuroimage, 19(3), 1085-1094. doi: 10.1016/s10538119(03)00113-7

Butman, J. (2001). La cognición social y la corteza cerebral. Revista Neurológica Argentina, 26, 117-122.

Chicharro, J., Pérez-García, A., \& Sanjuán, P. (2011). Respuesta emocional en adictos a sustancias en tratamiento ambulatorio. Adicciones, 24(1), 243-246. doi: 10.20882/adicciones.118

Damasio, A. R., Tranel, D., \& Damasio, H. (1990). Individuals with sociopathic behavior caused by frontal damage fail to respond autonomically to social stimuli. Behavioral Brain Research, 41(2), 81-94. doi: 10.1016/0166-4328(90)90144-4

Dapretto, M., Davies, M. S., Pfeifer, J. H., Scott, A. A., Sigman, M., \& Lacobini, M. (2005). Understanding emotions in others: mirror neuron dysfunction in children with autism spectrum disorders. Nature Neuroscience, 9(1), 28-30. doi: 10.1038/nn1611

Ersche, K. D., Hagan, C. C., Smith, D. G., Jones, P. S., Calder, A. J., \& Williams, G. B. (2015). In the face of threat: neural and endocrine correlates of impaired facial emotion recognition in cocaine dependence. Translational psychiatry, 5(5). doi:10.1038/ tp.2015.58

Eslinger, P. J., \& Damasio, A. R. (1985). Severe disturbance of higher cognition after bilateral frontal lobe ablation: patient EVR. Neurology, 35(12), 1731-1741. doi: 10.1212/wnl.35.12.1731

España, L. (2013). La cognición social en el daño cerebral traumático. Propuesta de intervención. España: Institutt Guttmann. Recuperado de http://www.hela03.es/wp-content/uploads/2013/09/ Trabajo_LauraEspa\%C3\%B1a.pdf

Fernández-Serrano, M., Moreno, L., Pérez, M., \& Verdejo, A. (2012). Inteligencia emocional en individuos dependientes de cocaína. Trastornos adictivos, 14(1), 27-33. doi: 10.1016/s1575-0973(12) 70040-5

Fernández-Serrano, M. J., Pérez, M., \& Verdejo, A. (2011). What are the specific vs. generalized effects of drugs of abuse on neuropsychological performance? Neuroscience Biobehavioral Review, 35(3), 377-406. doi: 10.1016/j.neubiorev.2010.04.008

Fernández-Serrano, M., Lozano, O., Pérez-García, M., \& Verdejo-García, A. (2010) Impact of severity of drug use on discrete emotions recognition in polysubstance abusers. Drug and Alcohol Dependence, 109(1-3). doi: 10.1016/j.drugalcdep.2009.12.007.

Foisy, M., Kornreich, C., Petiau, C., Pérez, A., Hanak, C., \& Verbanck, P. (2007). Impaired emotional facial expression recognition in alcoholics: are these deficits specific to emotional cues? Psychiatry Research, 150(1), 33-41. doi: 10.1016/j. psychres.2005.12.008

Gil, R. (2007). Neuropsicología de la Cognición Social. En A. Gil, (Ed.). Manual de Neuropsicología (pp. 332-338). España: Elsevier Goldman, M. (1990). Experience-dependent neuropsychological recovery and the treatment of chronic alcoholism. Neuropsychology Review, 1(1), 75-101. doi: 10.1007/bf01108859 
Goldstein, R., \& Volkow, N. (2002). Drug addiction and its underlying neurobiological basis: neuroimaging evidende for the involvement of the frontal cortex. American Journal of Psychiatry, 159(10), 1642-1652. doi: 10.1176/appi.ajp.159.10.1642

Green, M., Penn, D., Bentall, R., Carpenter, W., Gaebal, W., Gur, R., ... Heinssen, R. (2008). Social cognition in schizophrenia: an NIMH workshop on definitions, assessment and research opportunities. Schizophrenia Bulletin, 34(6), 1211-1220. doi: 10. 1093/schbul/sbm145

Kemmis, L., Hall, J., Kingston, R., \& Morgan, M. (2007). Impaired fear recognition in regular recreational cocaine users. Psychopharmacology, 194(2), 151-159. doi: 10.1007/s00213-0070829-5

Kim, Y., Kwon, D., \& Chang, Y. (2011). Impairments of facial emotion recognition and theory of mind in methamphetamine abusers. Psychiatry Research, 186(1), 80-84. doi: 10.1016/j. psychres.2010.06.027

Kornreich, C., Blairy, S., Philippot, P., Hess, U., Noel, X., \& Streel, E. (2001). Deficits in recognition of emotional facial expression are still present in alcoholics after mid-to long-term abstinence. Journal of Studies on Alcohol and Drugs, 62(4), 533-542. doi: 10.15288/jsa.2001.62.533

Kornreich, C., Phillippot, P., Foisy, M., Blairy, S., Raynaud, R., Dan, B., ... Velbanck, P. (2002). Impaired emotional facial expression recognition is associated with interpersonal problems in alcoholism. Alcohol and alcoholism, 37(4), 394-400. doi: 10.1093/ alcalc/37.4.394

Kuypers, K., Steenberger, L., Toennes, S., \& Ramaekers, J. (2015). Emotion recognition during cocaine intoxication. European Neuropsychopharmacology, 25(11), 1914-1921. doi: 10.1016/j.euroneuro.2015.08.012

Martín-Contero, M., Secades-Villa, R., \& Tirapu-Ustárroz, J. (2012) Cognición social en adictos a opiáceos. Revista de Neurología, 55(12), 705-712

Maurage, F., Timary, P., Tecco, J., Lechantre, S., \& Samson, D. (2015). Theory of mind difficulties in patients with alcohol dependence: beyond the prefrontal cortex dysfunction hypothesis. Alcoholism: Clinical and Experimental Research, 39(6), 980-988. doi: 10.1111/acer.12717.

Pascual, B., Aguado, G., \& Sotillo, M. (2006). Aproximación a las diferentes perspectivas sobre la Teoría de la Mente. Revista de logopedia, foniatría y audiología, 26(3), 154-165. doi: 10.1016/ S0214-4603(06)70208-X
Rodríguez, S., \& Nute, L. (2013). Reinserción social de usuarios de drogas en rehabilitación. Una revisión bibliográfica. Revista Electrónica de Psicología Iztacala, 16(1), 172-196. Recuperado de http://www.iztacala.unam.mx/carreras/psicologia/psiclin/ vol16num1/Vol16No1Art12.pdf

Rosenberg, N. L., Grigsby, J., Dreisbachb, J., Busenbark, D., \& Grigsby, P. (2002). Neuropsychologic impairment and MRI abnormalities associated with chronic solvent abuse. Journal of Toxicology. Clinical Toxicology, 4O(1), 21-34.

Roser, P., Lissek, S., Tegenthoff, M., Nicolas, V., Juckel, G., \& Brüne, M. (2012). Alterations of theory of mind network activation in chronic cannabis users. Schizophrenia Research, 139(1-3), 1626. doi: 10.1016/j.schres.2012.05.020

Rowe, D. L., Cooper, N. J., Liddell, B. J., Clark, C. R., Gordon, E., \& Williams, L. M. (2007). Brain structure and function correlates of general and social cognition. Journal of Integrative Neuroscience, 6(1), 35-74. doi:10.1002/brb3.76

Salo, R., Ursu, S., Buonocore, M. H., Leamon, M. H., \& Carter, C. (2009). Impaired prefrontal cortical function and disrupted adaptive cognitive control in methamphetamine abusers: a functional magnetic resonance imaging study. Biological Psychiatry, 65(8), 706-709. doi: 10.1016/j.biopsych.2008.11.026

Sugranyes, G., Kyriakopoulos, M., Corrigall, R., Taylor, E., \& Frangou, S. (2011). Autism spectrum disorders and schizophrenia: meta-analysis of the neural correlates of social cognition. PLOS ONE, 6(10), 1-13. doi: 10.1371/journal.pone.0025322

Tirapu-Ustárroz, G., Pérez-Sayes, G., Erekatxo-Bilbao, M., \& Pelegrín-Valero, C. (2007). ¿Qué es la Teoría de la Mente? Revista de Neurología, 44(8), 479-489.

Townshend, J., \& Duka, T. (2002). Mixed emotions: alcoholic's impairments in the recognition of specific emotional facial expressions. Neuropsychology, 41(7), 773-782. doi: 10.1016/S00283932(02)00284-1

Vik, P., Cellucci, T., Jarchow, A., \& Hedt, J. (2010). Cognitive impairment in substance abuse. The Psychiatric Clinics of North America, 27(1), 97-109. doi: 10.1016/S0193-953X(03)00110-2

Weinstein, C. S., \& Shaffer, H. J. (1993). Neurocognitive aspects of substance abuse treatment: a psychotherapist's primer. Psychotherapy, 30(2), 317-333. doi: 10.1037/0033-3204.30.2.317

Woicik, P., Moeller, S., \& Alia-Klein, M. (2009). The neuropsychology of cocaine addiction: recent cocaine use masks impairment. Neuropsychopharmacology, 34(5), 1112-1122. doi: 10.1038/ npp.2008.60 


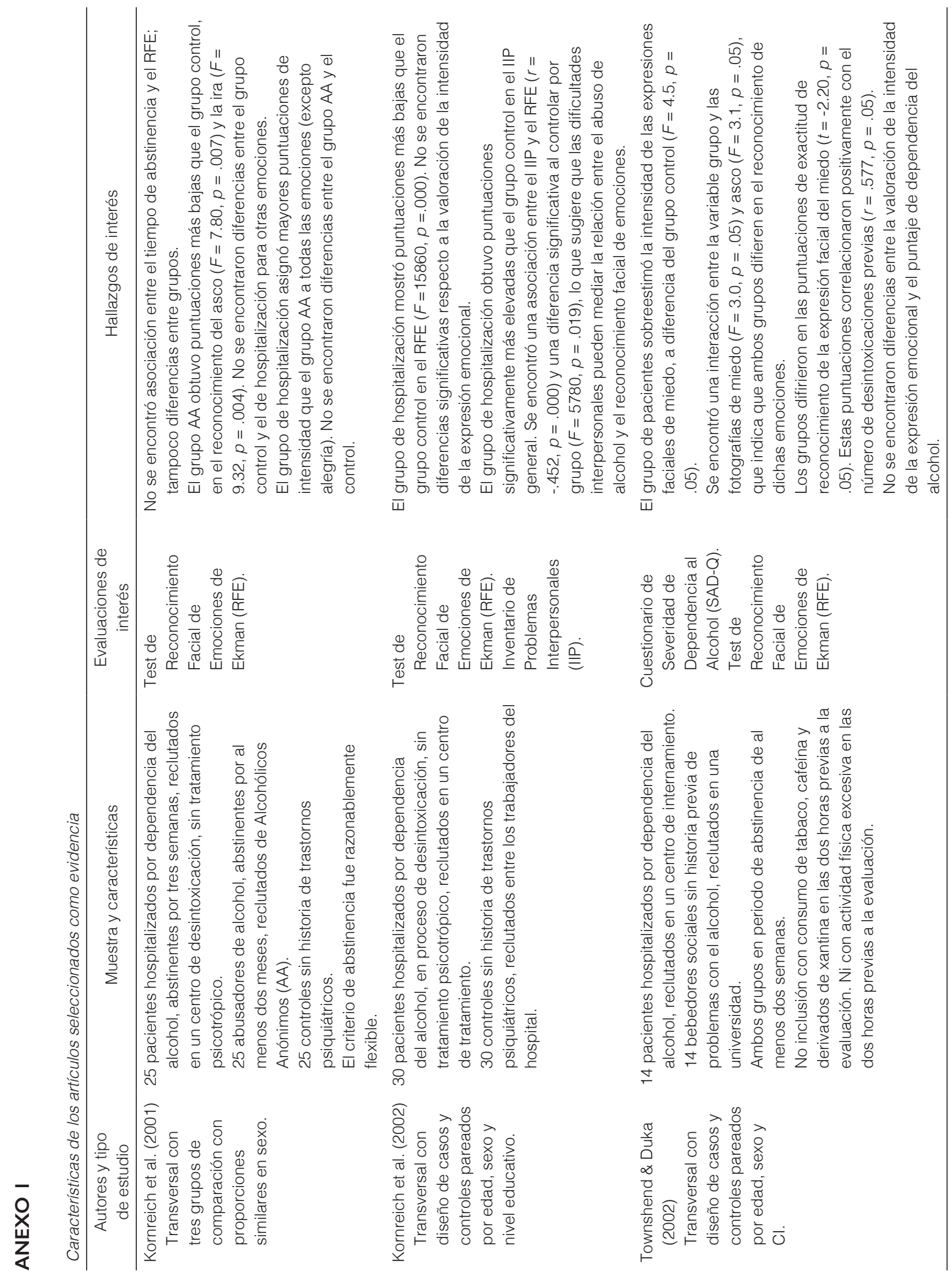




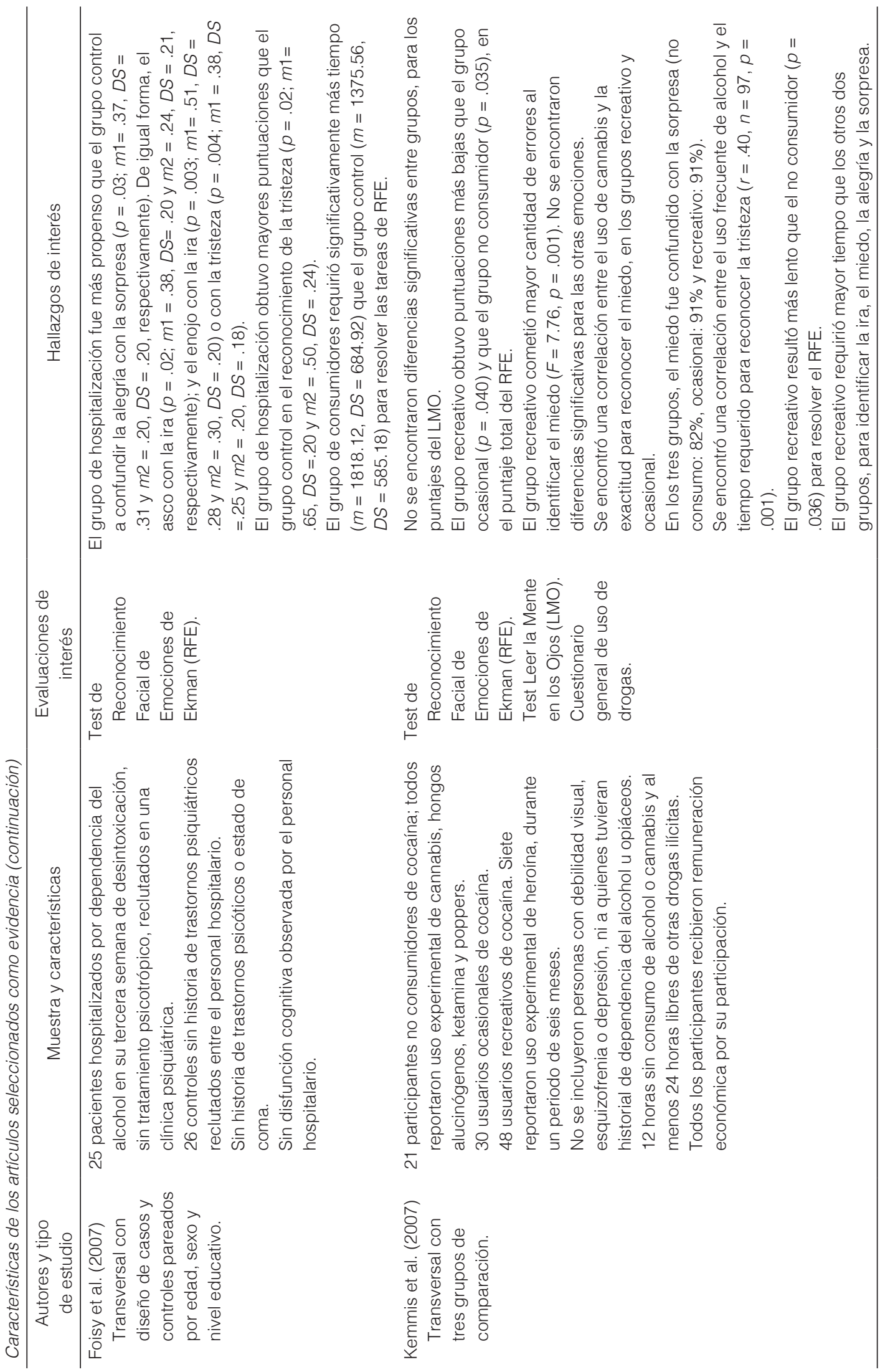




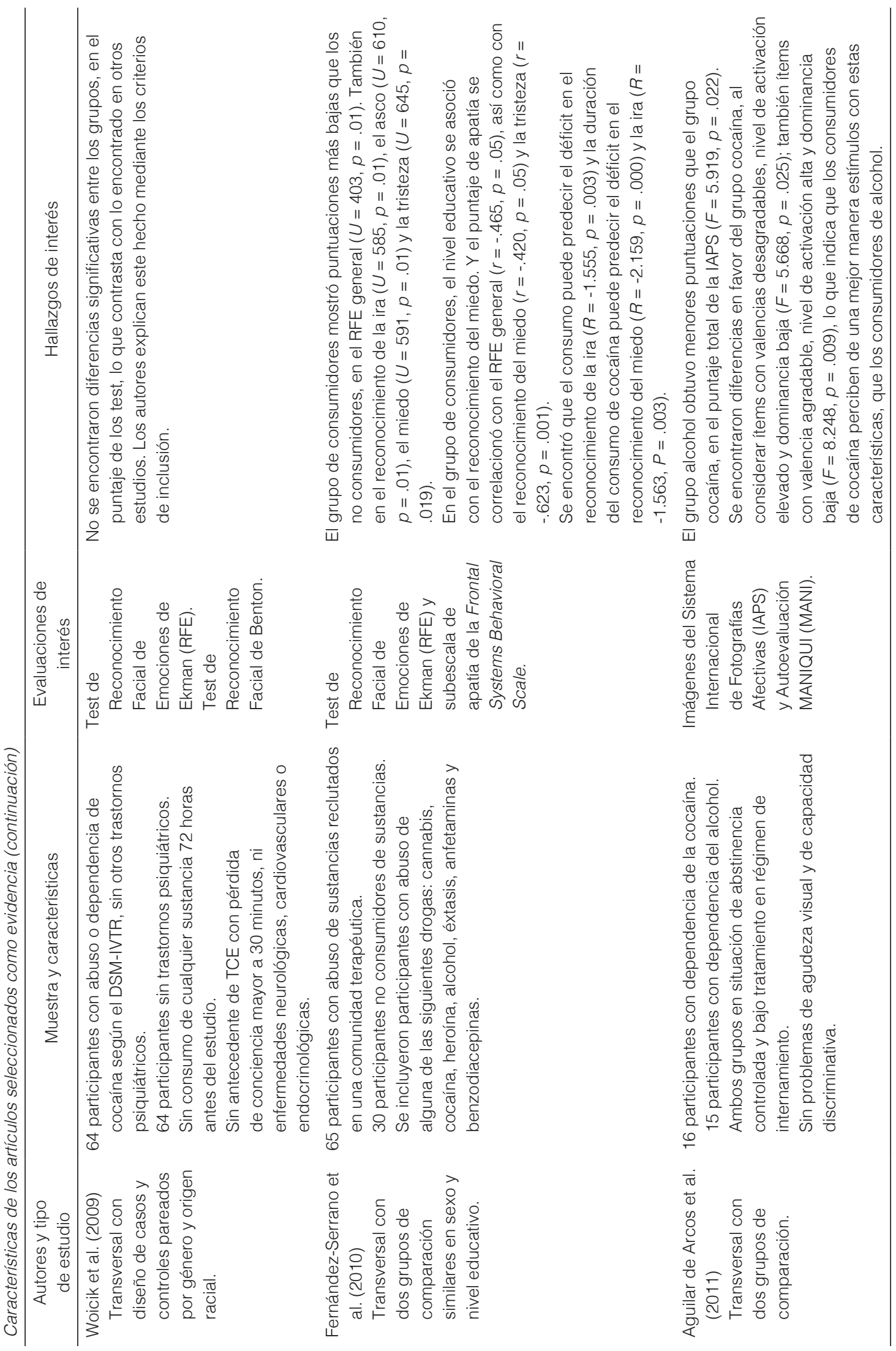




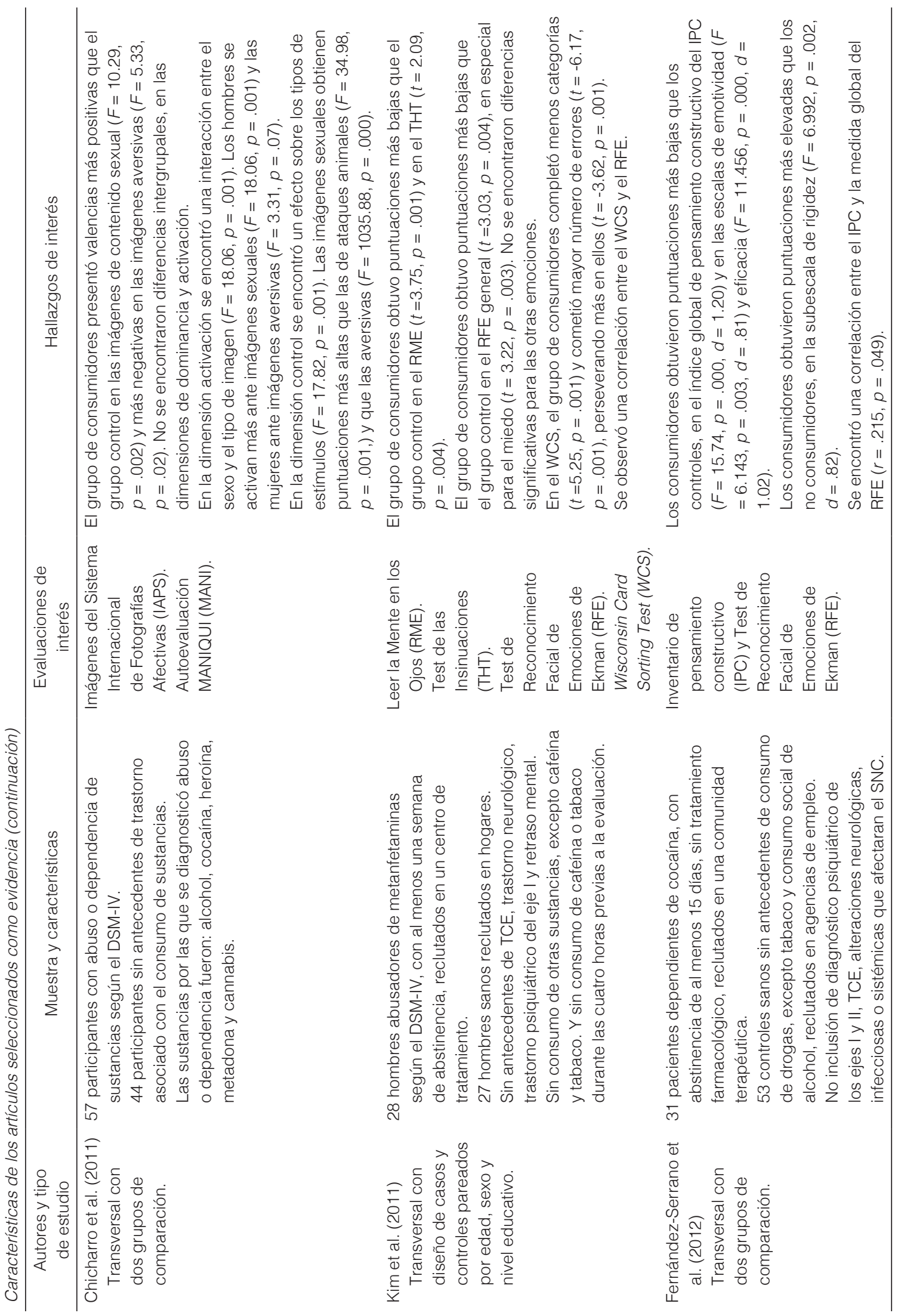




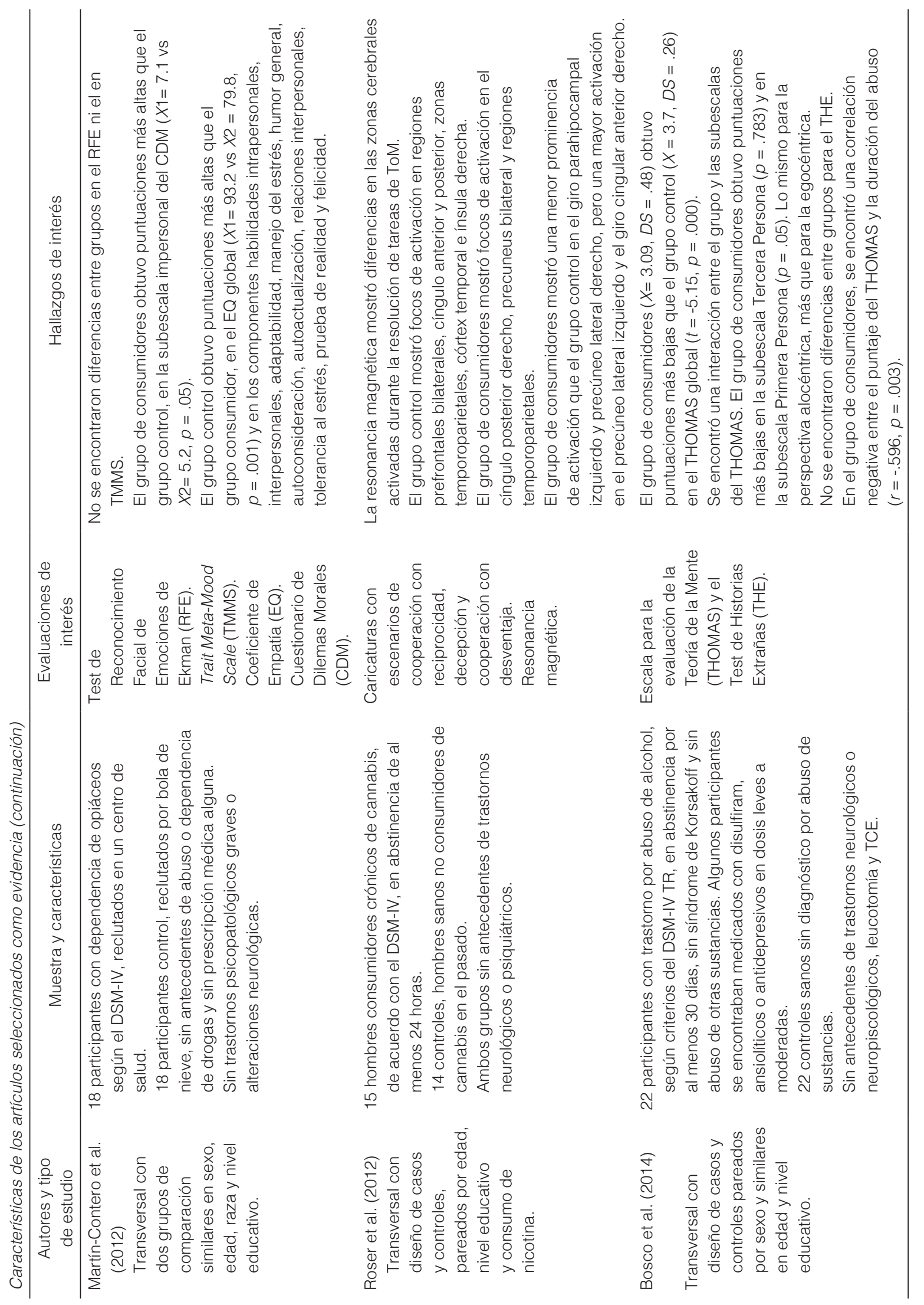




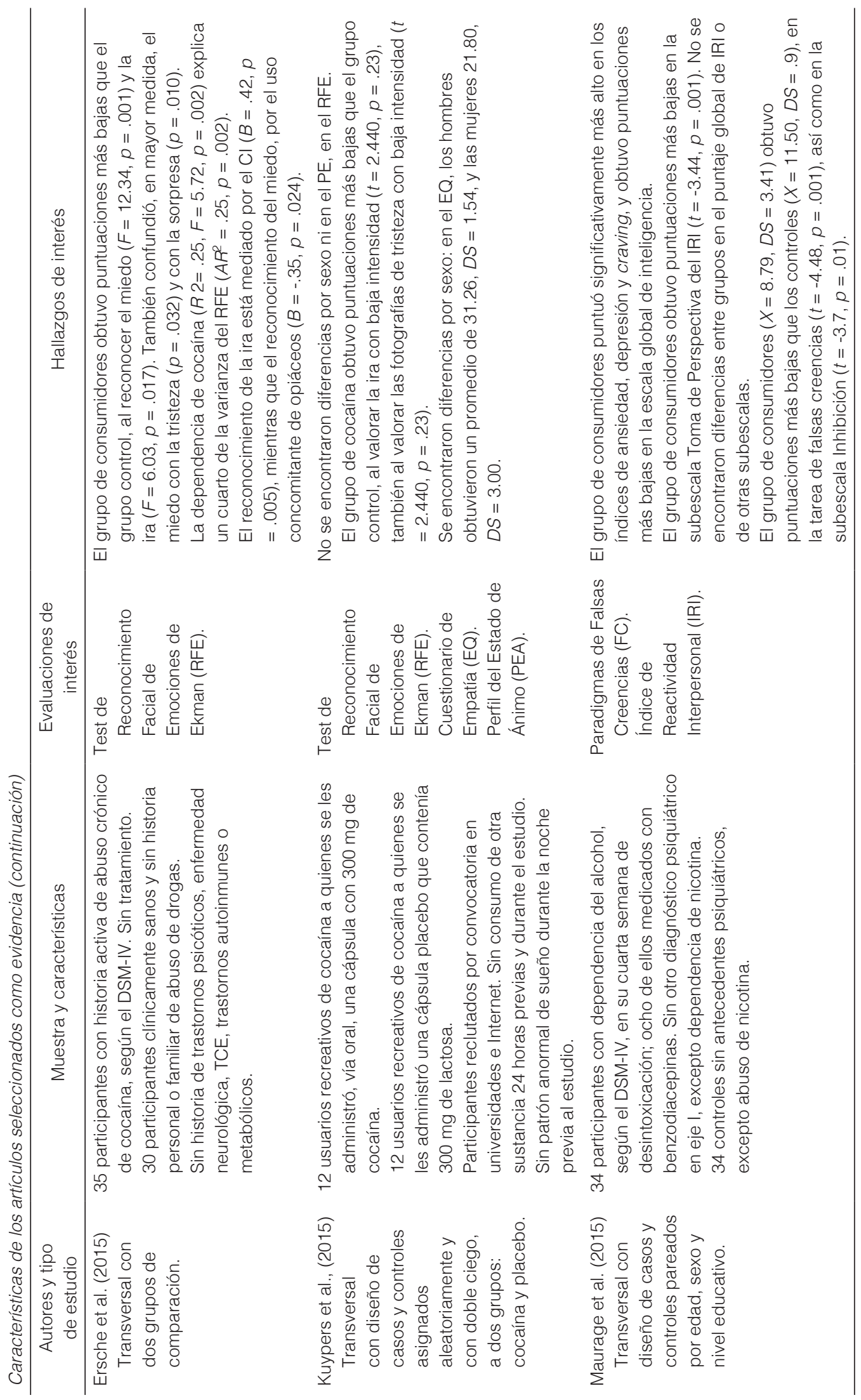

